\title{
Automatic Localization of the Carotid Wall in Ultrasound Imaging Based on Convolutional Neural Network
}

\author{
$1^{\text {st }}$ Alexandre G. Silva \\ $2^{\text {nd }}$ Eryk K. da Cruz \\ School of Technology \\ Federal University of Santa Catarina \\ Florianópolis, Brazil \\ alexandre.goncalves.silva@ufsc.br
}

\author{
$3^{\text {rd }}$ Rangel Arthur \\ $4^{\text {th }}$ Giulliano P. Carnielli \\ $5^{\text {th }}$ Henri A. de Godoy \\ Faculty of Technology \\ University of Campinas \\ Limeira, Brazil \\ rangel@ft.unicamp.br
}

\author{
$6^{\text {th }}$ Wilson N. Junior \\ $7^{\text {th }}$ Luís F. R. dos S. C. Romano \\ Faculty of Medical Sciences \\ University of Campinas \\ Campinas, Brazil \\ wilnj@fcm.unicamp.br
}

\begin{abstract}
Atherosclerosis is the leading cause of death in the world. It is a cardiovascular disease characterized by the accumulation of inflammatory cells and lipids inside the artery walls. In Brazil, more than $30 \%$ of all deaths are due to cardiovascular diseases. The carotid intima-media thickness, obtained from ultrasound images, may be an early estimate of atherosclerosis. This test is fast, safe and non-invasive, as well as being reproducible and relatively inexpensive. In this context, this work, based on convolutional neural networks and techniques of mathematical morphology, consists in automatically locating the region that covers the intima and media sublayers of carotid arteries. The proposed method obtained a score of $88 \%$ considering the trained model applied to 234 ultrasonographic images in two different datasets. The analysis of the neighborhood of the points obtained can be useful in the evaluation of cardiovascular risk factors.

Index Terms - carotid wall, ultrasound imaging, convolutional network, mathematical morphology
\end{abstract}

\section{INTRODUÇÃO}

A doença cardiovascular aterosclerose é a principal causa de morte no Brasil [19] e no mundo ${ }^{1}$, sendo caracterizada pelo acúmulo de lipídeos e célula inflamatórias dentro das paredes de artérias de médio e grande calibre [9], [21]. O exame de ultrassonografia na região do pescoço para determinação da Espessura Íntima-Média (EIM) carotídea pode ser um marcador precoce à aterosclerose, com a vantagem de ter custo relativamento baixo, ser rápido, seguro, não-invasivo e reprodutível. Porém seu significado clínico ainda é desconhecido [11]. Neste contexto, este trabalho, com base em algoritmos de aprendizagem de máquina e técnicas de morfologia matemática, consiste em automaticamente localizar uma ou mais regiões que contemplem as subcamadas íntima e média de artérias carótidas, de modo a servir como um referencial a processamentos seguintes que relacionem medidas e texturas a fatores de risco cardiovascular e marcadores de inflamação relacionados à aterosclerose. Indivíduos com aterosclerose subclínica (sem sintomas) devem ser preferencialmente identificados em um

\footnotetext{
${ }^{1}$ Top 10 causes of death worldwide (January 2017): http://www.who.int/mediacentre/factsheets/fs310/en/
}

estágio inicial, de modo que medidas preventivas possam ser iniciadas [1]. O desenvolvimento de aterosclerose ocorre durante décadas, e tem-se considerado que um dos primeiros estágios detectáveis de aterosclerose é o espessamento da parede arterial [25]. Os primeiros resultados de boa correlação entre duas linhas ecogênicas paralelas, da ultrassonografia bidimensional (modo-B), em tempo real, com a EIM aferida ao exame patológico, foram descritos [16]. Subsequentemente, diversos laboratórios validaram o uso do ultrassom para avaliar a EIM e demonstraram que esta medida é altamente reprodutível [7]. Vários estudos transversais demonstraram associações entre a EIM da artéria carótida comum e fatores de risco cardiovascular, como hipertensão arterial, tabagismo, hipercolesterolemia, diabetes e obesidade [12], [18]. De maneira similar, aumentos na EIM carotídea se associam com a presença de doença cardiovascular aterosclerótica [7]. Além disto, a EIM carotídea é um preditor de doença aterosclerótica coronária e de eventos coronários e cerebrovasculares [7], [25]. Estima-se que cada aumento de $0,1 \mathrm{~mm}$ na EIM carotídea se associa com elevações de $15 \%$ e $17 \%$ no risco de infarto do miocárdio e acidente vascular cerebral, respectivamente [25]. Com base nestes dados e por causa do fácil acesso anatômico, relativo baixo custo, reprodutibilidade e rapidez do exame, a EIM carotídea avaliada por ultrassonografia bidimensional é o exame não invasivo mais utilizado como medida de aterosclerose na prática clínica e em estudos científicos. A Figura 1 exemplifica esquematicamente uma artéria carótida normal (à esquerda) e outra com acúmulo de placa (à direita), e respectivas camadas "íntima" (mais interna), "média" (central) e "adventícia" (mais externa). Observa-se que a EIM é relativamente maior no segundo caso, podendo indicar obstrução parcial ou total do fluxo sanguíneo pelo "lúmen". A EIM carotídea mensurada pela ultrassonografia bidimensional é o exame mais utilizado para estimar a aterosclerose subclínica na prática clínica e em pesquisa. É um procedimento nãoinvasivo, de fácil acesso anatômico, relativo baixo custo, reprodutível e de rápida execução. Porém diversos estudos 
têm indicado que esta medida não é um marcador específico de aterosclerose, particularmente por não discriminar entre um aumento da espessura da parede arterial causada por uma hipertrofia da camada média ou por doença aterosclerótica.

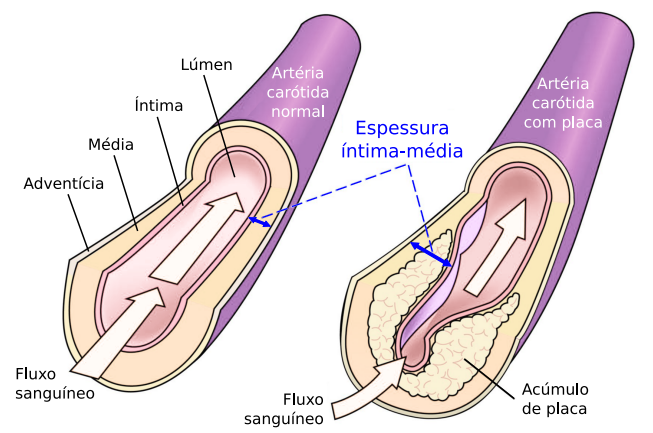

Figura 1: Subcamadas íntima, média e adventícia carotídea, e EIM para a artéria carótida normal e com comprometimento de fluxo sanguíneo pelo lúmen devido à placa. Figura adaptada de [17].

\section{MATERIAIS E MÉTODOS}

O grupo da FCM/UNICAMP demonstrou recentemente ser capaz de separar e mensurar individualmente as camadas íntima e média carotídea por meio da ultrassonografia bidimensional, utilizando transdutores vasculares de alta resolução, com ótima acurácia e reprodutibilidade. Portanto, pode-se estimar a espessura da camada íntima arterial, a qual é considerada uma medida mais precoce e específica de aterosclerose, além da espessura da camada média. Contudo, o significado clínico destas medidas permanece desconhecido. Neste contexto, é preciso estabelecer relações entre a espessura de cada subcamada carotídea (íntima e média) e os principais fatores de risco cardiovascular, tais como hipertensão, diabetes, tabagismo, hipercolesterolemia e obesidade. Também é necessário avaliar a relação entre as subcamadas e biomarcadores de inflamação e estresse oxidativo [5]. Um sistema totalmente automatizado de medidas e caracterização da carótida, por meio de descritores de imagem, pode contribuir significativamente no sentido de sugerir respostas a algumas destas importantes e desconhecidas relações. Antes, porém, espera-se que a localização das paredes dos vasos também seja automatizada e robusta.

A incapacidade de os exames ultrassonográficos convencionais discriminarem as camadas íntima e média se deve principalmente às características do transdutor vascular utilizado para medir a artéria carótida. A avaliação carotídea bidimensional convencional utiliza transdutores vasculares com frequências em torno de 8-10 MHz, que permitem identificar as duas interfaces arteriais (lúmen-íntima e média-adventícia), mas não são capazes de identificar adequadamente a interface entre as camadas íntima e média, conforme é mostrado na Figura 2 (à esquerda). Para superar esta limitação, o grupo de pesquisa da UNICAMP, que possui grande experiência em estudos analisando a EIM carotídea por ultrassonografia convencional em diversas situações clínicas, dedicou-se recentemente a desenvolver metodologias voltadas para a obtenção de imagens ultrassonográficas carotídeas bidimensionais de alta resolução que permitam a identificação mais precisa da interface entre as camadas íntima e média. Neste contexto, foi observada que a análise da parede carotídea utilizando transdutores vasculares de alta frequência (10-14 MHz) permite a obtenção de imagens mais acuradas da estrutura arterial, identificando com maior precisão os limites das camadas íntima e média e, principalmente, a interface entre estas camadas, de acordo com a Figura 2 (à direita).

Utilizando transdutor vascular de alta frequência (10-14 $\mathrm{MHz}$ ), a equipe publicou recentemente um estudo piloto que identificou e mensurou as espessuras das camadas íntima e média da artéria carótida comum de 41 indivíduos aparentemente saudáveis, não obesos e com idade média de 63 anos [22]. Nesta amostra, foi evidenciado que a EIM é um fenótipo determinado principalmente pela camada média, e não pela camada íntima (Figuras 1 e 2). Não menos importante, constatou-se que as medidas das camadas foram bastante reprodutíveis. Estes dados sugerem que a EIM parece ser uma boa medida de hipertrofia da camada média, mas não necessariamente da camada íntima, e consequentemente, da aterosclerose. Como a aterosclerose é uma doença inflamatória [9], [21], estes últimos achados dão suporte adicional à ideia de que o espessamento da camada íntima seja uma medida mais apropriada da carga aterosclerótica. Por outro lado, a associação da EIM e espessura da camada média com medidas de adiposidade abdominal reforçam a ideia de que a EIM é uma medida de fenótipo vascular adverso, o que pode contribuir para explicar sua maior associação com eventos cardiovasculares reportadas em estudos epidemiológicos [25].
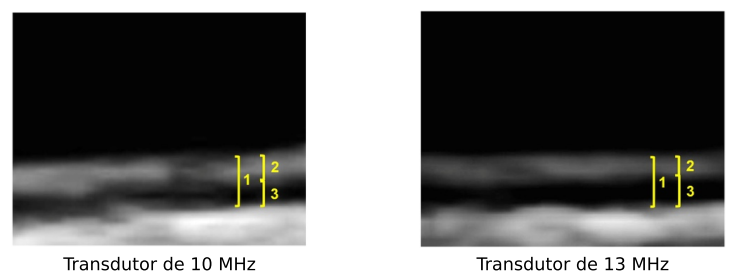

Figura 2: Imagens da espessura "íntima-média" (1), "íntima" (2) e "média" (3) de artéria carótida, obtidas por meio de ultrassonografia bidimensional, utilizando transdutor a $10 \mathrm{MHz}$ (à equerda) e a 13 MHz (à direita) [11].

\section{A. População de estudo e aquisição de imagens}

Este trabalho utiliza duas bases de imagens particulares da UNICAMP, ambas com cada amostra em $636 \times 422$ pixels. A primeira com 46 amostras obtidas de 11 indivíduos, e a segunda com 188 amostras de 23 indivíduos. A aquisição foi aprovada pelo Comitê de Ética em Pesquisa da UNICAMP (CAAE:56841616.5.0000.5404). Todos os indivíduos têm idade $>18$ anos e $<80$ anos, e assinaram o termo de Consentimento Livre e Esclarecido. Os tamanhos amostrais são estimados com base em estudos prévios [2], [22], [23], 
[26]. As artérias carótidas serão avaliadas por meio de um aparelho de ecodoppler Vivid Q (General Electric, USA) equipado com um transdutor vascular de alta frequência (10-14 MHz), conforme descrito previamente [22]. São obtidas imagens das artérias carótidas comuns (sendo da carótida esquerda e da carótida direita) $2 \mathrm{~cm}$ próximas à região do bulbo carotídeo. Em cada imagem, são traçadas cinco medidas manuais da espessura da camada íntima, da espessura da camada média, e da EIM, utilizando o software ImageJ (U.S. National Institutes of Health, Maryland, USA), conforme descrito previamente [22]. A localização das medidas manuais, feitas por especialistas, são usadas como ground-truth para avaliar a qualidade dos resultados computacionais. Havendo centenas ou milhares de imagens a analisar, qualquer assistência ou atuação humana acaba se tornando um processo oneroso e caro. $\mathrm{O}$ objetivo do trabalho não é o de substituir o trabalho de um especialista na tarefa de localizar as artérias, mas de pré-classificar e caracterizar as imagens, de maneira mais confiável possível e totalmente automática, para a posterior avaliação médica.

\section{TRABALHOS RELACIONADOS}

A técnica de Francesco et al. [6] é baseada em uma filtragem e detecção de bordas, seguida por uma heurística de busca de máximos locais dos perfis de cada coluna da imagem, remoção de pontos isolados, e caracterização de duas linhas por regressão, de modo que se possam identificar as interfaces "lúmen-íntima" e "média-adventícia". O procedimento é repetido dentro de uma região de interesse selecionada manualmente em vídeo, até que o usuário considere a medida calculada adequada. Molinari et al. [14] comparam quatro métodos completamente automáticos para a medição da EIM (denominadas CALEX, CAMES, CARES e CAUDLES) e um semiautomático (denominado FOAM), e apresentam sua experiência usando estes algoritmos para estimação de borda de "lúmen-íntima" e "média-adventícia". São relatadas quatro diferentes abordagens: baseada em realce de borda, baseada em treinamento de máquina, baseada em extração de características, baseada em fluxo de borda direcional. Petroudi et al. [15] apresentam um solução baseada em contornos ativos, e conjunto de nível (level set) de contornos ativos sem bordas, para segmentar diferentes regiões da carótida de lúmen e parede carotídea. O método de Chaudhry et al. [3] consiste no alinhamento de imagens, separação de objetos de interesse e processamento morfológico, segmentação da artéria baseada em contorno ativo, medida da EIM, e classificação usando SVM (support vector machine), entre normal e não-normal, das imagens segmentadas. Ilea et al. [10], por sua vez, efetuam detecção automática da região de interesse (ROI), filtragem de bordas em multiresolução, modelo de seleção de EIM, e reconstrução de arestas. Neste trabalho, é realizado um rastreamento (tracking) da EIM em quadros de vídeo, durante o ciclo cardíaco completo. Loizou et al. [13] se baseiam no método de segmentação por snakes (contornos ativos), filtros usando estatísticas de primeira ordem (tais como variância e média) de vizinhanças de pixel (janela $5 \times 5$ ), sendo descrito como um modelo de ruído multiplicativo. Por fim, modelam a classificação de doença cardiovascular (diferença entre normal e não-normal) usando redes SVM e RBF (Gaussian Radial Basis Function). Zhao et al. [27] efetuam um pré-processamento com filtragem passa-baixa Gaussiana para atenuação de ruído e definição de uma ROI em cada quadro de vídeo. A aquisição de medidas de EIM é feita com base em snakes, diferentes morfologias e dinâmicas durante o ciclo cardíaco.

A proposta do presente trabalho é baseada em Rede Neural Convolucional ou Convolutional Neural Network (CNN), sendo vantajosa no sentido de ser generalista e robusta para variadas bases de dados. Outro benefício vem da possibilidade de melhoria da acurácia em função da aprendizagem ocorrer a partir do treinamento de um conjunto maior de imagens. Não foi identificado nenhum outro trabalho de localização das paredes de carótida totalmente automático usando tal abordagem. Deste modo, entende-se que a técnica pode significar uma contribuição nesta área. Espera-se, em especial, que este trabalho seja a primeira etapa para a posterior medida da EIM, com acurácia relativamente alta (idealmente > 90\%) independente da base e da quantidade de imagens utilizadas.

\section{AlgORITMO PROPOSTO}

A primeira etapa do algoritmo proposto consiste na modelagem e treinamento de uma CNN, a partir de recortes $100 \times 100$ pixels, em imagens escolhidas aleatoriamente (podendo haver dezenas desses recortes para uma mesma imagem), manualmente rotulados em duas classes (positivo como pertencente ou negativo se não pertencente à parede de um vaso), sendo a maior parte dos recortes usada para treinamento do modelo e quantidades menores para validação e teste. Ao todo tinhamse 400 recortes para treinamento (200 positivos e 200 negativos), sendo que $15 \%$ (60 recortes) desse total utilizado para validação, e o restante (340 recortes) para treinamento. Não é possível dizer, com exatidão, a quantidade de recortes positivos e negativos usados para treinamento e validação, já que as imagens para validação são selecionadas aleatoriamente pelo algoritmo. Os testes são realizados após o treinamento, em um conjunto de imagens inédito para a rede. Esse conjunto de teste contém 78 recortes (39 positivos e 39 negativos). A arquitetura da rede neural foi adaptada de uma parte da FCN U-Net [20], [24] e um esquema detalhado da rede neural utilizada pode ser visto na Figura 4. As adaptações foram feitas a fim de tentar encontrar um modelo que melhor se adaptasse aos objetivos deste trabalho. As adaptações incluem:

1) Substituição da segunda metade da rede U-Net por uma camada "Flatten", seguida de uma camada "Dense". Essa alteração se deve ao fato de que as camadas retiradas tinham como objetivo a segmentação, enquanto neste trabalho o objetivo da rede neural é apenas de classificação.

2) Utilização de uma função Sigmóide para ativação da camada "Dense" de saída, em conjunto com uma função de custo denominada "Binary Cross-Entropy" (Entropia Cruzada Binária), apropriadas a problemas de classificação binária. 
Este modelo suficientemente treinado de rede é gravado e utilizado em todas as 207 imagens em dois conjuntos de dados (banco A com 46 itens, e banco B com 188), conforme a Figura 3. Todos os detalhes da arquitetura da CNN, modelada em Keras sobre TensorFlow [8], podem ser vistos na Figura 4. Nos testes efetuados, a cada região $100 \times 100$ pixels da imagem original (aquisição em $636 \times 422$ pixels), obtida a cada passo de 10 pixels na horizontal e vertical, padroniza-se seu redimensionamento a $28 \times 28$ pixels para entrada na arquitetura da CNN. A dimensão de $28 \times 28$ pixels foi escolhida por ser a menor testada sem comprometer os resultados, tornando o processamento mais eficiente. Deste modo, cada pedaço $100 \times 100$ pixels da entrada, a CNN gera uma probabilidade de se tratar de uma "parede" ou "não parede" de vaso. Somente no caso de ser uma região de parede, essa probabilidade é adicionada a uma imagem resultante (inicialmente de zeros). Na Figura 3, o resultado desta acumulação é exibido na imagem do canto direito-inferior em pseudocores. Observa-se que a soma final é maior exatamente ao redor de pixels prováveis de serem parte de uma parede de vaso carotídeo. O último passo do algoritmo refere-se a um processamento morfológico sobre essa imagem acumuladora, dado pela seguinte sequência:

1) Limiarização (thresholding) a partir da seleção de valores iguais ou acima de $75 \%$ do máximo da imagem acumuladora [4], exemplificado pelos contornos em vermelho na imagem resultante final;

2) Afinamento morfológico (thinning) com supressão de pontos finais em 20 iterações [4], resultando na linha em verde da figura;

3) Seleção de ponto central por afinamento morfológico (thinning) usando supressão de pontos finais com padrão homotópico [4], exibido como ponto central em azul.

Para que o resultado seja bem sucedido, espera-se que este ponto central obtido, conforme exibido na imagem final da Figura 3 em azul, tenha alguma intersecção ou com a camada íntima ou com a camada média da carótida. De outro modo, contabiliza-se como erro de localização. A delimitação das camadas, para uso como ground-truth, foi feita manualmente por um especialista.

\section{Resultados}

A CNN da Figura 4 foi treinada com 340 recortes de imagem e testada com 78 recortes, sendo 39 positivos (referentes a uma parede de vaso) e 39 negativos (não pertencentes a uma parede carotídea). Foram realizadas diversas sessões de treino, inicialmente variando o número de épocas. Testes foram realizados para 100, 50, 30, 20 épocas, pois, como pode ser observado na Figura 5 (um gráfico gerado em uma das sessões de treinamento do modelo, que compara perda e acurácia de treino e validação), a perda de validação decresce, acompanhando a perda de treino durante um determinado número de épocas, para então começar a crescer; a acurácia de validação cresce no início, acompanhando a acurácia de treino, e então estabiliza ou começa a cair. Essa separação das curvas de treino e validação, onde a perda e acurácia de treino continuam melhorando e as de validação estabilizam ou

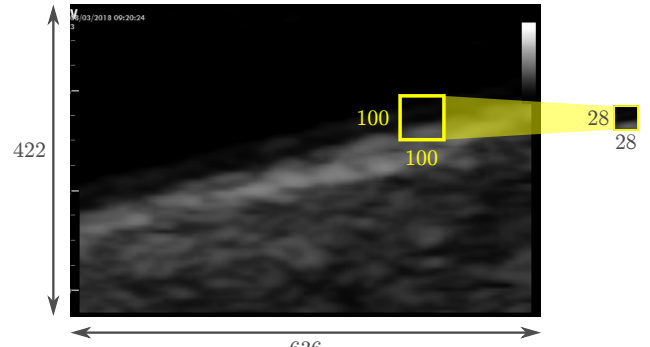

636
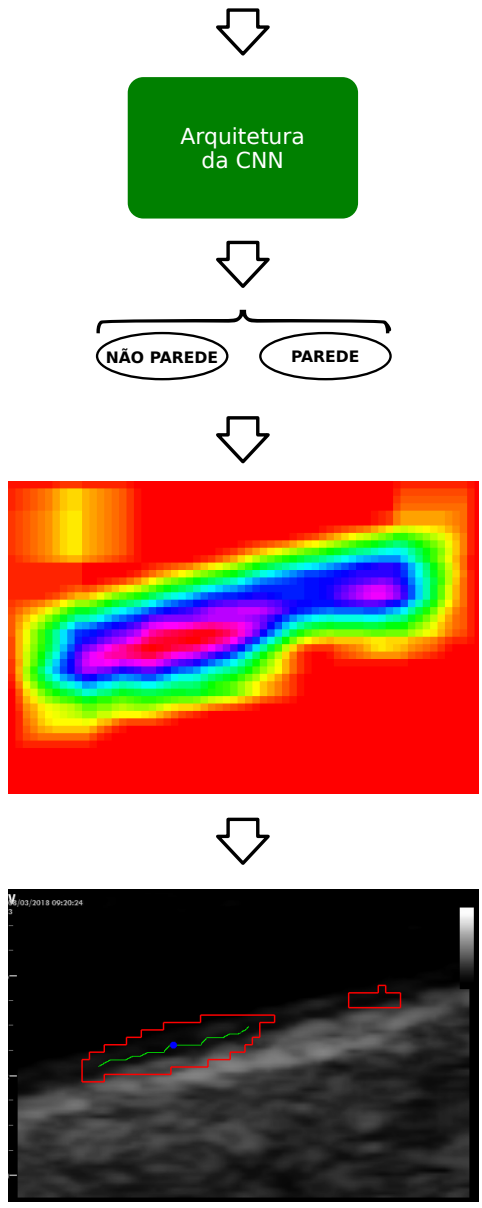

Figura 3: Algoritmo proposto.

tendem a piorar, indica um sobreajuste (overfitting) do modelo às imagens de treinamento. Chegou-se ao número de 20 épocas como o mais adequado, pois treina o modelo suficientemente bem, sem que ocorra sobreajuste. Após 20 épocas, a acurácia obtida foi de $93,5 \%$. Ou seja, dado um recorte de $100 \times 100$ de uma imagem completa de exame, é possível predizer com 93,5\% de certeza qual a sua classe, dentre duas possibilidades ("parede" ou "não parede").

Com todos os pesos da CNN ajustados, a predição foi aplicada a cada pedaço de cada imagem de exame, considerando passos de 10 pixels no deslocamento do janelamento nas duas direções (horizontal e vertical). Dois bancos de dados foram utilizados e os resultados da localização automática, em comparação com a observação humana, são apresentados na 


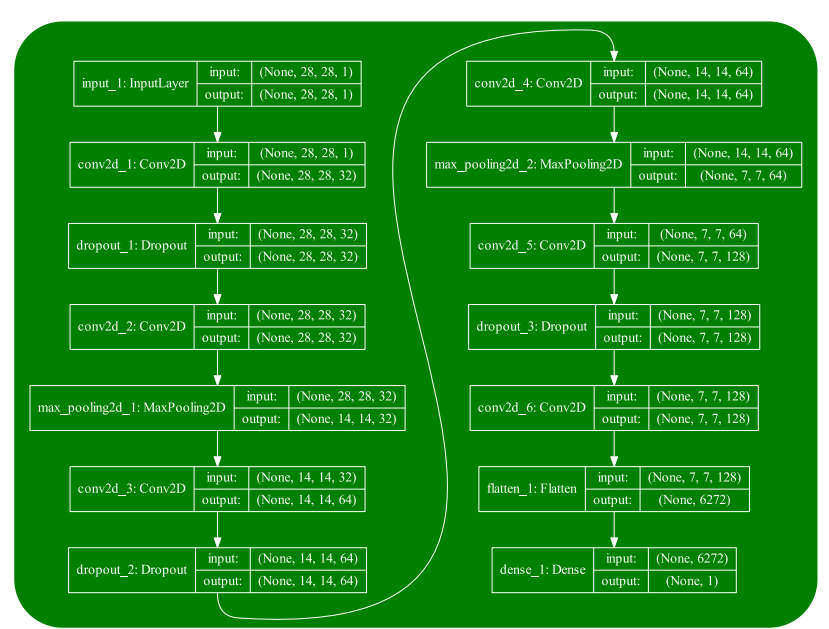

Figura 4: Arquitetura da CNN (Rede Neural Convolucional) modelada em Keras.
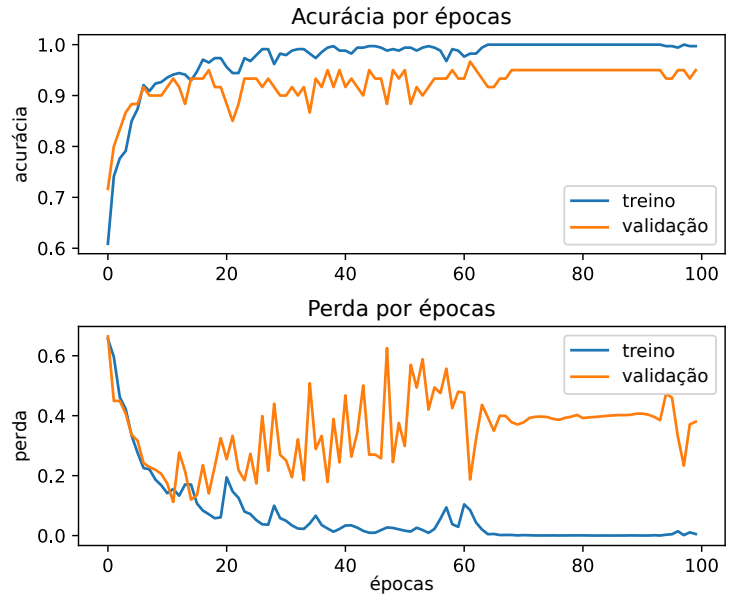

Figura 5: Acurácia e perda no treinamento e validação.

Tabela I.

Tabela I: Resultados da localização de paredes carotídeas.

\begin{tabular}{ccc}
\hline Conjuntos de imagens & Acertos & Erros \\
\hline Banco A & $43(93,48 \%)$ & $3(6,52 \%)$ \\
Banco B & $164(87,23 \%)$ & $24(12,77 \%)$ \\
TOTAL & $207(88,46 \%)$ & $27(11,54 \%)$ \\
\hline
\end{tabular}

Qualquer imperfeição da resposta do algoritmo não é considerada como acerto. Na Figura 6, um exemplo de erro de localização é ilustrado. Apesar de três pontos centrais de regiões, obtidos automaticamente após o afinamento dos componentes conexos gerados pela limiarização da imagem acumuladora (conforme explicado anteriormente), estarem sobre a subcamada "média" da carótida, um quarto ponto não apresenta qualquer intersecção com a subcamada "íntima" ou com a "média". O próximo passo do trabalho consiste em medidas automáticas de EIM. Na Figura 7, um caso bem sucedido de determinação de três medidas de EIM, para cada ponto central, é ilustrado. Este estudo está em andamento e ainda apresenta acurácia baixa.
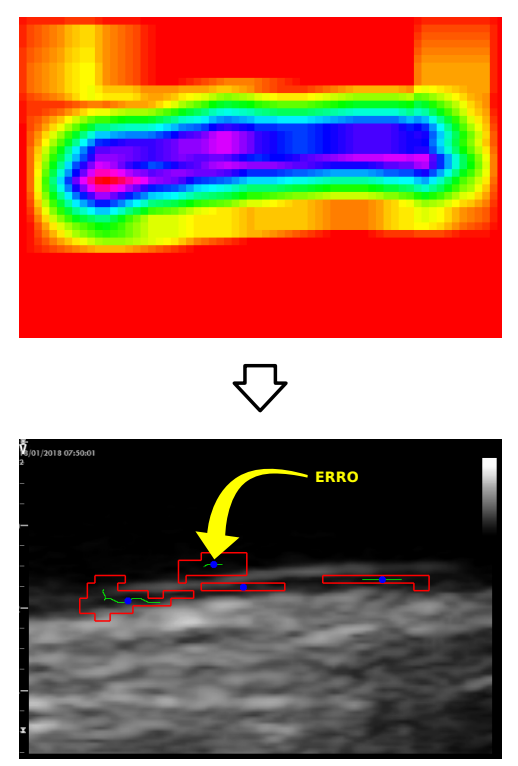

Figura 6: Exemplo de resultado considerado como erro de localização.

\section{CONCLUSÕES}

Este trabalho apresentou uma aplicação de CNN e morfologia matemática na área cardiovascular, concluindo a etapa inicial de localização para a resolução parcial do problema de medição e caracterização das subcamadas "íntima" e "média" carotídea, importantes na avaliação de fatores de risco cardiovascular. Apesar do pouco número de recortes usados no treinamento da rede, esta se mostrou robusta para duas diferentes bases de exames, com acerto de localização totalmente automática de parede de vaso acima de $88 \%$.

Como trabalho futuro, são necessários testes com outras configurações de arquitetura de rede, no sentido de caracterizar a solução como de aprendizagem profunda de máquina, envolvendo uma quantidade de recortes centenas ou milhares de vezes maior. Outro aspecto a ser refinado é o de pósprocessamento da imagem acumuladora. Na Figura 6, por exemplo, a coloração azulada da imagem acumuladora (acima) está perfeitamente sobre a parede carotídea, mas parte das regiões internas avermelhadas, não estão. Neste caso, a escolha de um limiar mais baixo provavelmente geraria uma única linha e ponto central sobre as subcamadas desejadas.

Assim que os pontos centrais de interesse, sobre a "íntimamédia", possam ser determinados de forma robusta, a etapa sequinte é a de caracterizar essas subcamadas, relacionando com eventuais diagnósticos para auxílio à avaliação médica.

\section{REFERÊNCIAS}

[1] Alawi A. Alsheikh-Ali, Georgios D. Kitsios, Ethan M. Balk, Joseph Lau, and Stanley Ip. The vulnerable atherosclerotic plaque: Scope of the literature. Annals of Internal Medicine, 153(6):387-395, 2010.

[2] Vera R. Bellinazzi, José A. Cipolli, Marcio V. Pimenta, Paula V. Guimar aes, and José A. Pio-Magalh aes; Otavio R. Coelho-Filho; Tor BieringSørensen; José R. Matos-Souza; Andrei C. Sposito; Wilson Nadruz. Carotid flow velocity/diameter ratio is a predictor of cardiovascular events in hypertensive patients. Journal of Hypertension, 33(10):20542060, 2015. 

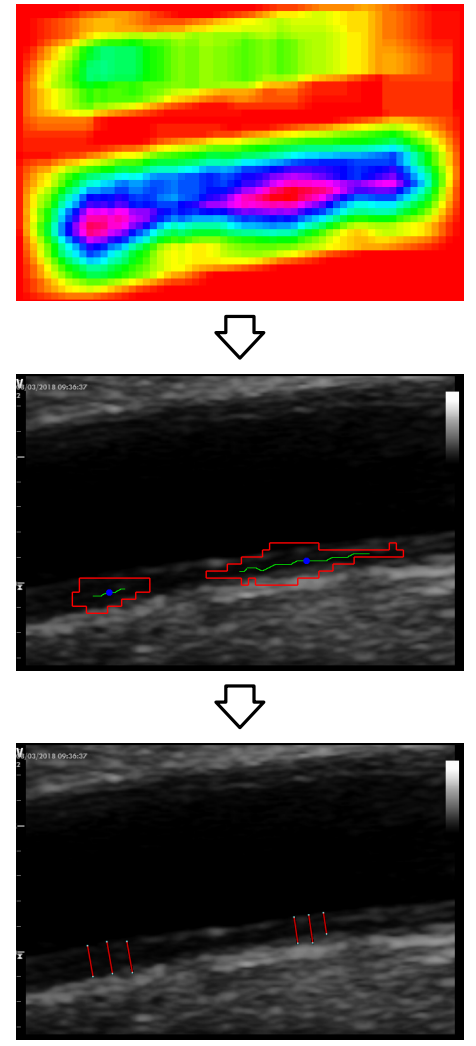

Figura 7: Exemplo de determinação automática de medidas de íntima-média em estudo em andamento.

[3] Asmatullah Chaudhry, Mehdi Hassan, Asifullah Khan, and Jin Young Kim. Automatic active contour-based segmentation and classification of carotid artery ultrasound images. Journal of Digital Imaging, 26(6):1071-1081, 2013.

[4] Edward R Dougherty and Roberto A Lotufo. Hands-on morphological image processing, volume 71. SPIE Press Bellingham, 2003.

[5] Belda Dursun, Evrim Dursun, Gultekin Suleymanlar, Beste Ozben, Irfan Capraz, Ali Apaydin, and Tomris Ozben. Carotid artery intimamedia thickness correlates with oxidative stress in chronic haemodialysis patients with accelerated atherosclerosis. Nephrology Dialysis Transplantation, 23(5):1697-1703, 2008.

[6] Faita Francesco, Gemignani Vincenzo, Bianchini Elisabetta, Giannarelli Chiara, Ghiadoni Lorenzo, and Demi Marcello. Real-time measurement system for evaluation of the carotid intima-media thickness with a robust edge operator. Journal of Ultrasound in Medicine, 27(9):1353-1361, 2008.

[7] Philip Greenland, Jonathan Abrams, Gerard P. Aurigemma, M. Gene Bond, Luther T. Clark, Michael H. Criqui, John R. Crouse, Lawrence Friedman, Valentin Fuster, David M. Herrington, Lewis H. Kuller, Paul M. Ridker, William C. Roberts, William Stanford, Neil Stone, H. Jeremy Swan, Kathryn A. Taubert, and Lewis Wexler. Prevention conference v: Beyond secondary prevention: identifying the high-risk patient for primary prevention: noninvasive tests of atherosclerotic burden: Writing group iii. Circulation, 101(1):E16-22, 2000.

[8] Antonio Gulli and Sujit Pal. Deep Learning with Keras. Packt Publishing, 2017.

[9] Göran K. Hansson. Inflammation, atherosclerosis, and coronary artery disease. The New England Journal of Medicine, 352(16):1685-1695, 2005.

[10] Dana E. Ilea, Caoimhe Duffy, Liam Kavanagh, Alice Stanton, and Paul F. Whelan. Fully automated segmentation and tracking of the intima media thickness in ultrasound video sequences of the common carotid artery. IEEE Transactions on Ultrasonics, Ferroelectrics, and Frequency Control, 60(1):158-177, 2013.

[11] Wilson Nadruz Junior. Relação entre subcamadas da parede carotídea, risco cardiovascular e micro RNAs em indivíduos brasileiros. Projeto de pesquisa apresentado ao CNPq para obtenção de Bolsa de Produtividade em Pesquisa, 2017.

[12] Sina Kianoush, Mohammad Yawar Yakoob, Mahmoud Al-Rifai, Andrew P. DeFilippis, Marcio S. Bittencourt, Bruce B. Duncan, Isabela M. Bensenor, Aruni Bhatnagar, Paulo A. Lotufo, and Michael J. Blaha. Associations of cigarette smoking with subclinical inflammation and atherosclerosis: ELSA-Brasil (The Brazilian Longitudinal Study of Adult Health). Journal of the American Heart Association, 6(6), 2017.

[13] Christos P. Loizou, Andrew Nicolaides, Efthyvoulos Kyriacou, Niki Georghiou, Maura Griffin, and Constantinos S. Pattichis. A comparison of ultrasound intima-media thickness measurements of the left and right common carotid artery. IEEE Journal of Translational Engineering in Health and Medicine, 3:(1900410)1-10, 2015.

[14] Filippo Molinari, Kristen M. Meiburger, Luca Saba, U. Rajendra Acharya, Giuseppe Ledda, Guang Zeng, Sin Yee Stella Ho, Anil T. Ahuja, Suzanne C. Ho, Andrew Nicolaides, and Jasjit S. Suri. Ultrasound IMT measurement on a multi-ethnic and multi-institutional database: our review and experience using four fully automated and one semiautomated methods. Computer Methods and Programs in Biomedicine, 108(3):946-960, 2012.

[15] Styliani Petroudi, Christos Loizou, Marios Pantziaris, and Constantinos Pattichis. Segmentation of the common carotid intima-media complex in ultrasound images using active contours. IEEE Transactions on Biomedical Engineering, 59(11):3060-3069, 2012.

[16] Paolo Pignoli, Elena Tremoli, Andrea Poli, Pierluigi Oreste, and Rodolfo Paoletti. Intimal plus medial thickness of the arterial wall: a direct measurement with ultrasound imaging. Circulation, 74(6):1399-406, 1986.

[17] Pufa. Higher dha, not epa, associated with lower carotid thickness in japanese men, December 2011. https://www.fatsoflife.com/cardiovascularhealth/higher-dha-not-epa-associated-with-lower-carotid-thickness-injapanese-men-2/.

[18] Baoge Qu and Tao Qu. Causes of changes in carotid intima-media thickness: a literature review. Cardiovascular Ultrasound, page 13:46, 2015.

[19] Antonio Luiz P. Ribeiro, Bruce B. Duncan, Luisa C. C. Brant, Paulo A. Lotufo, José Geraldo Mill, and Sandhi M. Barreto. Cardiovascular health in brazil: trends and perspectives. Circulation, 133(4):422-433, 2016.

[20] O. Ronneberger, P.Fischer, and T. Brox. U-net: Convolutional networks for biomedical image segmentation. In Medical Image Computing and Computer-Assisted Intervention (MICCAI), volume 9351 of LNCS, pages 234-241. Springer, 2015.

[21] Russell Ross. Atherosclerosis - an inflammatory disease. The New England Journal of Medicine, 340(2):115-126, 1999.

[22] Amanda V. Sardeli, Arthur F. Gáspari, Guilherme de Rossi, Giovana V. de Souza, Thiago M.F. de Souza, Cláudia R. Cavaglieri, José R. MatosSouza, Wilson Nadruz Jr., and Mara P.T. Chacon-Mikahil. Carotid intima-media thickness is associated with media rather than intima thickness. Atherosclerosis, 261:169-171, 2017.

[23] R. Schreiber, A. V. Campos-Coelho, L. Brandão, R. L. Guimarães, A. J. Kamada, M. C. Ferreira-Sae, J. R. Matos-Souza, J. A. Cipolli, J. L. de Lima-Filho, S. Crovella, and W. Nadruz. Mannose-binding lectin (mbl2) polymorphisms and inflammation in hypertensive patients. International Journal of Immunogenetics, 38(6):525-527, 2011.

[24] Evan Shelhamer, Jonathan Long, and Trevor Darrell. Fully convolutional networks for semantic segmentation. IEEE Trans. Pattern Anal. Mach. Intell., 39(4):640-651, April 2017.

[25] Stijn C.H. van den Oord, Eric J.G. Sijbrands, Gerrit L. ten Kate, David van Klaveren, Ron T. van Domburg, Antonius F.W. van der Steen, and Arend F.L. Schinkel. Carotid intima-media thickness for cardiovascular risk assessment: systematic review and meta-analysis. Atherosclerosis, 228(1):1-11, 2013.

[26] Patrick Yerly, Pedro Marquès-Vidal, Reza Owlya, Eric Eeckhout, Lukas Kappenberger, Roger Darioli, and Michèle Depairon. The atherosclerosis burden score (ABS): a convenient ultrasound-based score of peripheral atherosclerosis for coronary artery disease prediction. Journal of Cardiovascular Translational Research, 8(2):138-147, 2015.

[27] Shen Zhao, Zhifan Gao, Heye Zhang, Yaoqin Xie, Jianwen Luo, Dhanjoo Ghista, Zhanghong Wei, Xiaojun Bi, Huahua Xiong, Chenchu Xu, and Shuo Li. Robust segmentation of intima-media borders with different morphologies and dynamics during the cardiac cycle. IEEE Journal of Biomedical and Health Informatics, PP(99):1-11, 2017. 